F. Alvi

A. Kumar

A.D. Clayson

Received: 23 June 2007

Accepted: 26 September 2007

Published online: 21 December 2007

F. Alvi • A. Kumar • A.D. Clayson

North-West Pelvic and Acetabular Surgical

Unit, Department of Orthopaedics

North Manchester General Hospital

Manchester, UK

A. Kumar (两)

170 Woodhouse Lane

Sale, Cheshire M33 4LN, UK

E-mail: arun_orth@yahoo.co.uk

\section{Open reduction and internal fixation of an unstable pelvic ring injury during pregnancy}

\begin{abstract}
We report a case of an unstable vertical shear injury of the pelvis in a pregnant patient with a viable foetus. The combination of skeletal traction and pelvic external fixation failed to achieve satisfactory reduction. Open reduction and internal fixation of the pubic symphysis and the left sacro-iliac joint were performed without affecting the pregnancy. At the one-year followup, the patient was completely asymptomatic. We feel that surgical treatment of pelvic fractures can be offered to pregnant patients after
\end{abstract}

thoroughly considering the potential benefits and dangers of surgery to the patient and the foetus.

\section{Introduction}

Vertical shear injuries are unstable injuries resulting from disruption of both anterior and posterior components of the pelvic ring. Severe pelvic injuries during pregnancy not only threaten an existing pregnancy but also affect subsequent pregnancy and parturition [1]. Inadequate treatment of unstable and displaced pelvic injuries can result in significant long-term residual disability [2]. We report a case of an unstable vertical shear injury to the left hemipelvis in a 12-week pregnant patient who was treated by open reduction and internal fixation.

\section{Case report}

A 17-year-old girl was brought to the accident and emergency department following a motor vehicle accident. She was a passenger of a motorcycle which collided with a motorcar. On arrival she was fully conscious and haemodynamically stable but complained of pain in the left hip region. It was noted that she was approximately 12 weeks pregnant. This was confirmed by ultrasonography, which showed a viable foetus with no injuries. After initial resuscitation, she was suspected of having an unstable injury to the left hemipelvis with disruption of the symphysis pubis and left sacroiliac joint (Fig. 1). There were no head, chest or abdominal injuries.

The patient was taken to theatre the same day for stabilisation of the unstable pelvic injury and to allow an obstetrician to perform a perineal examination under anaesthesia. The left hemipelvis was found to be vertically and rotationally unstable and was therefore temporarily stabilised by an anterior AO external fixator through Schanz pins inserted in the anterior iliac crest and skeletal traction through a distal femoral pin. On perineal examination, no obvious injuries to the genito-urinary tract were found. The patient remained stable and the foetus was 


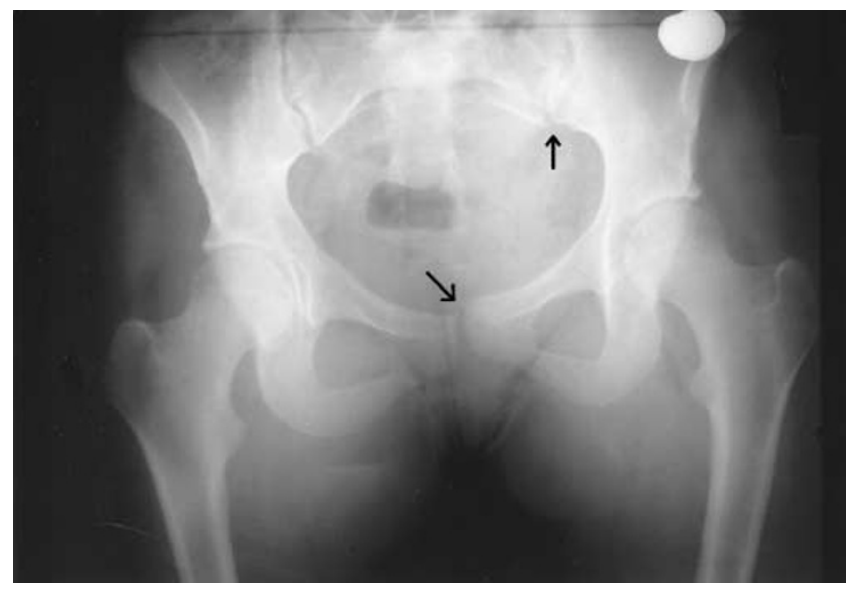

Fig. 1 Radiograph of the pelvis showing disruption of the pubic symphysis and the left sacro-iliac joint (arrows)

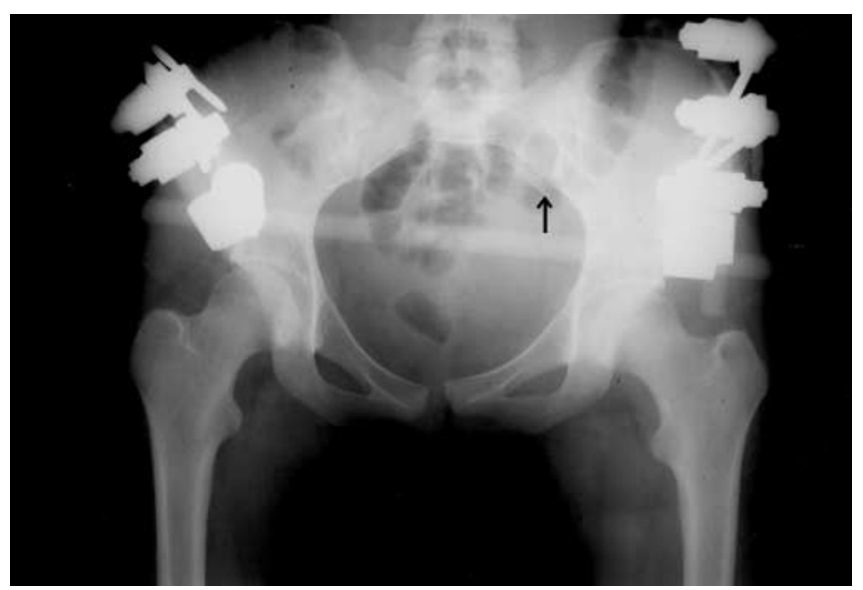

Fig. 2 Radiograph of the pelvis after application of an anterior external fixator, confirming vertical shear injury of the left hemipelvis with persistent displacement of the pubic symphysis and the left sacroiliac joint (arrow)

viable. Radiograph of the pelvis after application of the external fixator showed persistent vertical displacement of the left hemipelvis (Fig. 2). Considering the nature of the injury and displacement, the patient was considered at a significant risk of developing residual pain, long-term disability and shortening and also obstetric complications during the existing and future pregnancies. Therefore, potential benefits of open reduction and internal fixation like adequate reduction, early mobilisation and rehabilitation versus the risks of surgery to the pregnancy and the well-being of the foetus were discussed with the patient.

After thorough discussion, the patient decided to proceed with internal fixation of the pelvis. This was achieved by plating of the symphysis pubis through a Pfenensteil approach and plating of the left sacroiliac joint through the superior part of an anterior ilioinguinal approach. At the time of operation, the left hemipelvis was found displaced superiorly and posteriorly and multidirectional instability of the

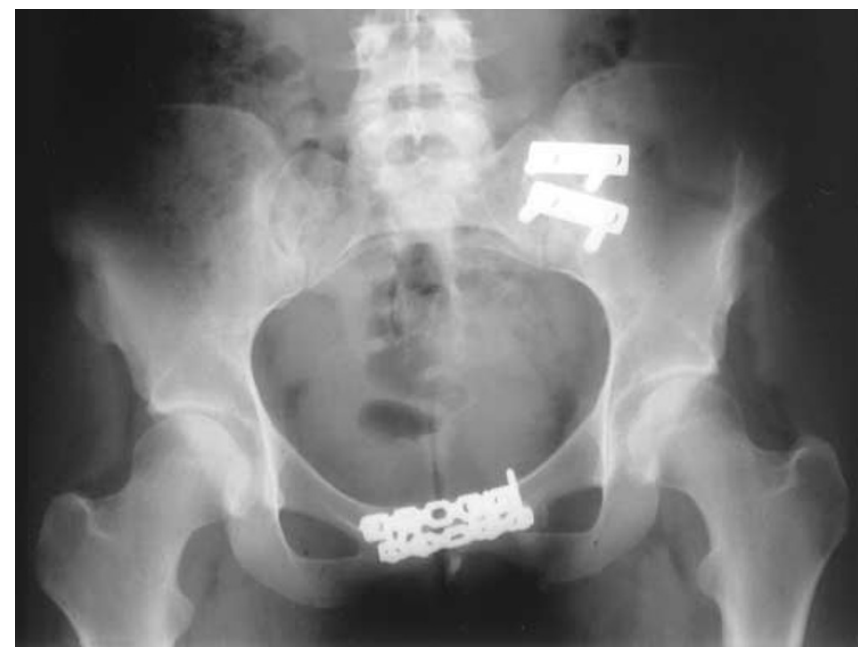

Fig. 3 Radiograph of the pelvis 12 months after internal fixation, showing good reduction of the left hemipelvis

pelvis was confirmed. Postoperatively, the patient made a satisfactory recovery and the foetus remained viable. The patient started mobilising without weight bearing on the left leg with the help of crutches. However, for unrelated personal reasons, the patient subsequently requested termination of pregnancy. Therefore, three weeks after surgery (15 weeks gestation), although the foetus was still viable, medical termination of the pregnancy was performed without complications. At about twelve months of follow-up, the patient was completely asymptomatic and was mobilising without any support; radiographs showed no loss of reduction (Fig. 3).

\section{Discussion}

Pelvic fracture in females can adversely affect genito-urinary, sexual and reproductive functions [3]. During pregnancy, pelvic fractures result in up to $35 \%$ incidence of foetal loss and a high incidence (37\%) of caesarean section in subsequent pregnancies [1]. Foetal loss can be either due to direct foetal injury, abruptio placenta or altered physiology of gestation resulting from severe trauma [4]. Increased incidence of caesarean section in subsequent pregnancy is usually due to an obstetrically inadequate pelvis resulting from displaced fractures and malunion [1]. Additionally, inadequate treatment of vertically shear injuries is associated with a high incidence of disability with associated pain, gait abnormality and limb length inequality due to a displaced hemipelvis [2]. The recent literature recommends internal fixation of unstable vertical shear injuries of the pelvis to achieve satisfactory functional results $[5,6]$. It also allows an early mobilisation and rehabilitation and avoids the risks of immobility associated with skeletal traction and anterior pelvic fixator. 
There is generally a trend to treat pregnant patients conservatively due to fear of loss of the foetus, but due to the paucity of information in the literature on surgical treatment of pelvic injuries during pregnancy, very little is known about the magnitude of associated risks and complications. Previously, internal fixation of an acetabular fracture in pregnancy has been reported without any adverse effect on the foetus in isolated case reports [7-9]. However, surgical management of pelvic fractures in pregnancy does pose a definite risk to the foetus. A review of the use of anaesthesia during pregnancy concluded that a normal foetus is able to withstand a variety of modern anaesthetic techniques and no currently used anaesthetics are known to be teratogenic. However, drugs like propofol, suxamethonium, isoflurane and nitrous oxide, which are rapidly eliminated by the mother and the neonate, should be used to minimise the exposure [10]. Other concern in such cases would be an increased risk of irradiation to the foetus. We did not perform computed tomography because of the radiation exposure and we do not think that the decision to operate led to any increase in radiation dose. In fact, we did not need to do serial check radiographs after the pelvis had been surgically stabilised. Our decision to treat this injury surgically was based on assessment of pelvic instability at the time of application of an external fixator and the demonstration of residual displacement in the radiograph after application of the pelvic fixator. The foetus survived through pelvic surgery and thereafter remained viable for three weeks. The other surgical option could have been to retain the anterior external fixator and fix the sacro-iliac joint with percutaneous screws. But retaining an external fixator for 3 months would have been quite cumbersome for a 12-week pregnant patient with added risk of pin track infection. Moreover, the use of percutaneous screws would have needed image intensifier and exposure to radiation in theatre.

We believe that surgical treatment of pelvic injuries during pregnancy with a viable foetus is possible after thoroughly considering the potential benefits and danger of surgery to the patient and the foetus. With adequate resuscitation and use of appropriate anaesthetic technique, pelvic fractures can be treated surgically during pregnancy. After initial resuscitation, such patients should be referred early to specialised units for advice and definitive management.

\section{References}

1. Speer DP, Peltier LF (1987) Pelvic fractures and pregnancy. J Trauma 12:474-480

2. Semba RT, Yasakawa K, Gustilo RB (1983) Critical analysis of results of 53 Malgaigne fractures of the pelvis. J Trauma 23:35-537

3. Copeland CE, Bosse MJ, McCarthy ML et al (1997) Effect of trauma and pelvic fracture on female genitourinary, sexu$\mathrm{al}$, and reproductive function. J Orthop Trauma 11:73-81

4. Ali J, Yeo A, Gara TJ et al (1997) Predictors of foetal mortality in pregnant trauma patients. J Trauma 42:782-785
5. Korovessis P, Baikousis A, Stamatakis $M$ et al (2000) Medium and long-term results of open reduction and internal fixation for unstable pelvic ring fractures. Orthopaedics 23:1165-1171

6. Ward EF, Tomasin J, Van der Griend RA (1987) Open reduction and internal fixation of vertical shear pelvic fractures. J Trauma 27:291-295

7. Dunlop DJ, McCahill JP, Blakemore ME (1997) Internal fixation of an acetabular fracture during pregnancy. Injury 28:481-482
8. Pals SD, Brown CW, Friermood TG (1992) Open reduction and internal fixation of an acetabular fracture during pregnancy. J Orthop Trauma 6:379-381

9. Yosipovitch Z, Goldberg I, Ventura E, Neri A (1992) Open reduction of acetabular fracture in pregnancy. A case report. Clin Orthop 282:229-232

10. Gin T (1993) Pharmacokinetic optimisation of general anaesthesia in pregnancy. Clin Pharmacokinet 25:59-70 